\title{
Nível de entendimento sobre prescrição farmacêutica no Brasil. Estamos preparados para essa nova realidade?
}

\author{
Understanding Pharmacist-prescribing role in Brazil. \\ Are we prepared for this new reality?
}

Recebido em: 29/06/2016 Aceito em: 24/08/2016
Ana Luiza Pelissari Pessanha de Paula SOARES'; Marco Antonio COSTA ${ }^{\mathbf{1}}$; Jorge Juarez Vieira TEIXEIRA ${ }^{\mathbf{2}}$ ${ }^{1}$ Departamento de Farmácia; ${ }^{2}$ Departamento de Análises Clínicas e Biomedicina. Universidade Estadual de Maringá (UEM). Av. Colombo, 5790, Jd Universitário, Maringá, PR. CEP 87020-900, Brasil. E-mail:aninhappps@gmail.com

\section{ABSTRACT}

This study aimed to verify the understanding of Pharmacist-prescribing role, by Pharmacists working in community and retail pharmacies in the city of Maringa, Parana, Brazil. The obtained data were analyzed by OpenEpi ${ }^{\circledR}$ program and Fisher's exact test. After interviewing 159 Pharmacists, the results showed that $99.4 \%$ of them knew about the Pharmacist-prescription meaning. Among them, 70.4\% have read the Federal Council of Pharmacy Resolutions $\mathrm{N}^{\mathrm{o}}$ $585 / 13$ and 586/13, and only $34.0 \%$ were currently performing this Pharmacist role. The association of predictor variables (gender, age, university attended, years of experience, type of graduation, specialization and role performed in the pharmacy) didn't show statistically significant difference in the outcome (act of performing or not the Pharmacistprescription role). It can be concluded that the Pharmacist-prescription role is well understood in theory, but, because is a new professional role in Brazil, it is incipient in practice.

Keywords: Pharmacist-prescription role, Pharmaceutical Care, Health Care

\section{RESUMO}

O estudo teve como objetivo verificar o entendimento, dos farmacêuticos que atuam em Farmácias e Drogarias do município de Maringá, Paraná, sobre prescrição farmacêutica. Os dados foram analisados pelo Programa OpenEpi ${ }^{\circledR}$ e teste Exato de Fisher. Os resultados referentes a 159 farmacêuticos mostraram que 99,4\% sabiam o que é prescrição farmacêutica, 70,4\% leram as Resoluções № 585/13 e 586/13 do Conselho Federal de Farmácia e apenas 34,0\% estavam prescrevendo. A associação das variáveis preditoras (sexo, faixa etária, universidade onde se formou, tempo de formado, tipo de formação, especialização e função que exerce na farmácia) não apresentou diferença estatisticamente significativa com o desfecho (ato de realizar ou não a prescrição farmacêutica). Pode-se concluir que a prescrição farmacêutica é bem entendida na sua teoria, entretanto é incipiente na prática por ser um assunto ainda novo em termos de legislação.

Palavras chave: prescrição farmacêutica, Atenção Farmacêutica, Saúde Pública. 


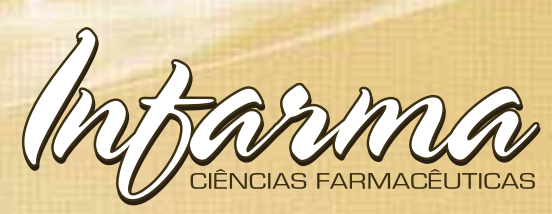

\section{INTRODUÇÃO}

A profissão farmacêutica está, historicamente, entre as mais antigas atividades profissionais que se desenvolveram como parte integrante da sociedade. E para compreender o atual cenário dos estudos farmacêuticos, é indispensável entender antecedentes históricos da profissão. No Brasil, os percursos foram marcados, principalmente, pela perda do mercado de trabalho para outros profissionais, como químicos, biomédicos, engenheiros químicos e de alimentos, devido à insegurança e ao despreparo dos profissionais da Farmácia durante a industrialização do medicamento. Outra parte importante desse cenário foi a mudança do currículo do curso para uma formação generalista que possibilitou a especialização de outros profissionais na área farmacêutica e, atualmente, a busca da "reprofissionalização" da profissão farmacêutica $(1,2)$.

A profissão farmacêutica no Brasil foi marcada por três períodos de mudanças, de acordo com as alterações sociopolítico-econômicas do país. O primeiro surgiu com a indústria farmacêutica, no qual o farmacêutico passou de boticário à função limitada de dispensador, alterando sua formação acadêmica. A Farmácia Clínica e a Atenção Farmacêutica foram responsáveis pelo segundo período de mudança, contribuindo para o resgate da função farmacêutica $(3,4)$. Por fim, acredita-se que a prescrição farmacêutica possa ser considerada a terceira onda de mudança, ou seja, uma maneira de "reprofissionalização" da profissão farmacêutica, como forma de reafirmar a importância deste profissional na sociedade.

Como forma de reafirmar o farmacêutico perante a sociedade e os outros profissionais, o Brasil vem apresentando modificações positivas no cenário desta profissão. Podem ser destacadas as Resoluções do $\mathrm{CFF} \mathrm{N}^{\circ} 585$, de 29 de agosto de 2013, que dispôs sobre as atribuições clínicas do farmacêutico e a $\mathrm{N}^{\circ} 586$, aprovada em 29 de agosto de 2013, que regulariza a prescrição farmacêutica no Brasil e fortaleceu os serviços farmacêuticos previstos na Resolução No 357/2001, para a melhora da qualidade da assistência à saúde. Essas medidas poderão contribuir, principalmente, com a diminuição diária de riscos causados pela automedicação e racionalização do uso do medicamento $(5,6,7)$.

Tradicionalmente, o ato de prescrever é associado aos profissionais médicos. No entanto, nas últimas décadas, mudanças na legislação vêm ocorrendo em vários países como Canadá, Nova Zelândia, Reino Unido e Estados Unidos, instituindo e regularizando a prescrição não-médica, ou seja, a extensão do direito de prescrever a profissionais como farmacêuticos, enfermeiros, odontólogos entre outros (8).

Sendo assim, o presente estudo teve como objetivo verificar o entendimento sobre prescrição farmacêutica dos farmacêuticos responsáveis por Farmácias e Drogarias do município de Maringá, Paraná.

\section{MÉTODOS}

Foi realizado um estudo de corte transversal no município de Maringá, PR, localizado na mesorregião Norte Central Paranaense, com população de 391.698 habitantes (9). Até 2014, o município contava com 232 farmácias/drogarias de acordo com dados fornecidos pelo Conselho Regional de Farmácia do Estado do Paraná (CRF-PR).

A fim de verificar o entendimento sobre prescrição farmacêutica, foram entrevistados farmacêuticos responsáveis pelas farmácias e drogarias de dispensação localizadas no Município de Maringá, Paraná, em dezembro do ano de 2014 e janeiro de 2015. Os endereços das farmácias foram disponibilizados pelo CRF-PR.

A população foi definida a partir da amostragem aleatória simples (10). Para isso foram consideradas todas as farmácias que constavam da lista fornecida pelo CRF-PR, sendo que apenas um farmacêutico de cada farmácia selecionada na amostra foi entrevistado.

A amostra foi calculada pelo Programa Epi-info versão 6.4 no módulo Statcalc. Considerando o erro de estimativa de $5 \%$ e a confiabilidade e precisão da amostra em $95 \%$, foi adotado o percentual de $50 \%$ que fornece o tamanho amostral máximo, sendo obtido, desta forma, o total final de farmacêuticos para a amostra. Foram acrescentados $20 \%$ ao número de farmacêuticos, levando em consideração eventuais perdas. Logo, a população amostral estimada foi de 175 (75,4\%) farmacêuticos. A partir do número amostral estipulado, foi realizada a seleção dos sujeitos de forma aleatória.

O critério de inclusão para participação do farmacêutico foi: ser farmacêutico auxiliar ou responsável técnico pela farmácia no Município de Maringá, PR. Como critérios de exclusão, foi estabelecido a não aceitação do Termo de Consentimento Livre e Esclarecido ou a mera recusa em participar.

Sendo assim, para a investigação do perfil dos farmacêuticos atuantes nas farmácias do município, um profissional farmacêutico de cada farmácia visitada foi 


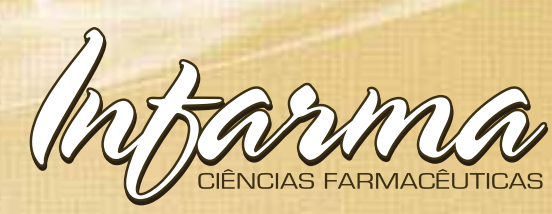

escolhido aleatoriamente para a entrevista independente de sua experiência, conhecimentos de outros cenários profissionais ou pós-graduações realizadas.

Tabela 1: Características dos farmacêuticos entrevistados responsáveis pelas farmácias de dispensação, Município de Maringá, PR, Brasil, no período de 12/2014 a 01/2015

\begin{tabular}{|c|c|c|}
\hline Variáveis preditoras & $\mathrm{N}$ & $\%$ \\
\hline \multicolumn{3}{|l|}{ Sexo } \\
\hline Masculino & 62 & 39,0 \\
\hline Feminino & 97 & 61,0 \\
\hline \multicolumn{3}{|l|}{ Faixa etária } \\
\hline $20-24$ & 11 & 6,9 \\
\hline $25-29$ & 59 & 37,1 \\
\hline $30-34$ & 31 & 19,5 \\
\hline $35-39$ & 23 & 14,5 \\
\hline $40-44$ & 06 & 3,8 \\
\hline $45-49$ & 12 & 7,5 \\
\hline $50-54$ & 10 & 6,3 \\
\hline $55-59$ & 05 & 3,1 \\
\hline 60 ou mais & 02 & 1,3 \\
\hline \multicolumn{3}{|c|}{ Universidade onde se formou } \\
\hline Pública & 46 & 28,9 \\
\hline Particular & 113 & 71,1 \\
\hline \multicolumn{3}{|c|}{ Tempo de formado (ano) } \\
\hline$<3$ & 35 & 22,0 \\
\hline $4-6$ & 42 & 26,4 \\
\hline 7 ou + & 82 & 51,6 \\
\hline \multicolumn{3}{|l|}{ Tipo de formação } \\
\hline Habilitação & 64 & 40,3 \\
\hline Generalista & 95 & 59,7 \\
\hline \multicolumn{3}{|l|}{ Especialização } \\
\hline Sim & 47 & 42,0 \\
\hline Não & 65 & 58,0 \\
\hline \multicolumn{3}{|l|}{ Função na farmácia } \\
\hline Farmacêutico auxiliar & 61 & 38,4 \\
\hline Farmacêutico responsável técnico & 98 & 61,6 \\
\hline
\end{tabular}

Para a realização da pesquisa foi construído um instrumento de coleta de dados, em forma de questionário ou entrevista semiestruturada, com questões abertas e fechadas. O instrumento foi estruturado em duas partes, sendo a primeira para identificação do farmacêutico com relação ao perfil sociodemográfico e a segunda para caracterização do conhecimento sobre prescrição farmacêutica.

A coleta de dados foi realizada por meio de visita à farmácia onde se encontrava o profission- al, conforme as variáveis preditoras (sexo, faixa etária, universidade onde se formou, tempo de formado, tipo de formação, especialização e função que exerce na farmácia) e de desfecho (realização ou não da prescrição farmacêutica).

Todos os profissionais concordantes com a pesquisa assinaram o termo de consentimento livre e esclarecido.

Os dados foram organizados em planilha no Microsoft Excel relativos às questões fechadas. As respostas das questões abertas foram agrupadas em categorias de acordo com as respectivas similaridades. Os resultados foram analisados em frequências simples e absolutas. O programa OpenEpi ${ }^{\circledR}$ foi utilizado para verificação de diferenças significativas. As análises estatísticas foram realizadas pelo Teste Exato de Fisher, com significância para $\mathrm{p}<0,05$.

O estudo foi aprovado pelo Comitê Permanente de Ética em Pesquisa Envolvendo Seres Humanos (COPEP) da Universidade Estadual de Maringá (Parecer 781.266).

\section{RESULTADOS E DISCUSSÃO}

A população foi constituída por 159 (90\%) dos 175 farmacêuticos responsáveis pelas farmácias de dispensação.

Um total de $10 \%$ dos farmacêuticos se recusaram a responder o questionário. As farmácias em que o farmacêutico não estava presente após três visitas foram substituídas, assim como as farmácias que não foram encontradas no endereço indicado.

Os dados da Tabela 1 permitiram identificar o predomínio de farmacêuticos do sexo feminino, 97 (61,0\%), e um número elevado de profissionais jovens, ou seja, 70 $(44,0 \%)$ se concentraram entre as idades de 20 a 29 anos.

Com relação à instituição de ensino, se pública ou privada, o estudo mostrou que $113(71,1 \%)$ profissionais fizeram o curso de Farmácia em universidades particulares, sendo que a maioria $(76,1 \%)$ em instituições de ensino de Maringá. Desses, 42 (26,4\%) cursaram na Universidade Estadual de Maringá (UEM), 41 (25,8\%) no Centro Universitário Cesumar (Unicesumar) e 38 (23,9\%) na Faculdade Ingá (Uningá). O município de Maringá conta atualmente com três cursos de graduação em Farmácia, sendo dois particulares e um público.

Foi possível observar, no perfil de formação dos profissionais, que $82(51,6 \%)$ farmacêuticos atuantes 


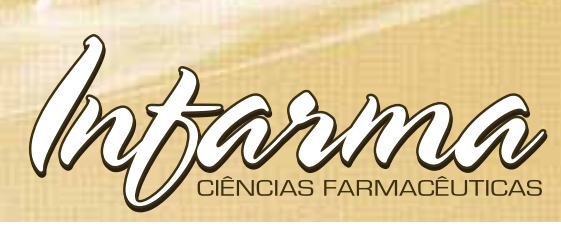

em farmácias de dispensação em Maringá já estavam formados há pelo menos 7 anos, sendo 95 (59,7\%) formados pelo currículo generalista, e apenas 47 (42,0\%) entrevistados alegaram ter especialização em áreas relacionadas à profissão farmacêutica. Ademais, 98 (61,6\%) exerciam o cargo de farmacêutico responsável técnico, atuando regularmente por 40 horas semanais nos estabelecimentos.
Em relação ao entendimento sobre prescrição farmacêutica, dos 159 farmacêuticos entrevistados, 96 $(60,4 \%)$ relataram saber completamente o que é a prescrição farmacêutica e $62(39,0 \%)$ conheciam parcialmente. Entretanto, dos 158 farmacêuticos que disseram "conhecer completamente ou parcialmente", 47 (29,6\%) afirmaram não terem lido as Resoluções № 585 e 586 de 2013 do CFF (Tabela 2).

Tabela 2: Perfil do entendimento dos farmacêuticos responsáveis pelas farmácias de dispensação em relação à prescrição farmacêutica, Município de Maringá, PR, Brasil no período de 12/2014 a 01/2015

\begin{tabular}{|c|c|c|c|}
\hline & Variáveis & $\mathbf{N}^{0}$ & $\%$ \\
\hline \multicolumn{4}{|c|}{ Conhecimento sobre prescrição farmacêutica $(n=159)$} \\
\hline Sim, completamente & & 96 & 60,4 \\
\hline Sim, parcialmente & & 62 & 39,0 \\
\hline Não & & 01 & 0,6 \\
\hline \multicolumn{4}{|c|}{ Leu as Resoluções* $(n=159)$} \\
\hline Sim & & 112 & 70,4 \\
\hline Não & & 47 & 29,6 \\
\hline \multicolumn{4}{|c|}{ Conhecimento sobre o que o farmacêutico pode prescrever $(n=112)$} \\
\hline MIPs & & 105 & 59,0 \\
\hline Fitoterápicos & & 50 & 28,0 \\
\hline Homeopáticos & & 23 & 13,0 \\
\hline \multicolumn{4}{|c|}{ Realiza a prescrição farmacêutica $(n=112)$} \\
\hline Sim & & 38 & 34,0 \\
\hline Não & & 74 & 66,0 \\
\hline \multicolumn{4}{|c|}{ Realizam o acompanhamento $(n=38)$ [farmacoterapêutico após a prescrição $(n=38)$ ] } \\
\hline Sim & & 28 & 73,7 \\
\hline Não & & 10 & 26,3 \\
\hline \multicolumn{4}{|c|}{ Atualização a respeito das classes $(n=159)$ [(farmacêuticas $(n=159)]$} \\
\hline $\operatorname{Sim}$ & & 119 & 74,8 \\
\hline Não & & 40 & 25,2 \\
\hline \multicolumn{4}{|c|}{ Estímulo a realizar a prescrição $(n=159)$ [farmacêutica $(n=159)$ ] } \\
\hline Sim & & 130 & 81,8 \\
\hline Não & & 29 & 18,2 \\
\hline
\end{tabular}

*Resolução 585/13 e 586/13. MIP = Medicamentos isentos de prescrição

Sobre o conhecimento do que o farmacêutico pode prescrever, dos 112 entrevistados que informaram ter lido essa resolução, $105(59,0 \%)$ sabiam que podiam prescrever os medicamentos isentos de prescrição (MIP), 50 (28,0\%) os fitoterápicos e 23 (13,0\%) os homeopáticos.

Outro aspecto analisado foi se os profissionais farmacêuticos estavam exercendo o ato de prescrever. Dos farmacêuticos que referiram ter conhecimento sobre o que o farmacêutico pode prescrever, apenas
38 (34,0\%) realizavam a prescrição farmacêutica; no entanto, a maioria $(73,7 \%)$ fazia acompanhamento dos pacientes. Ao considerar a forma como este é feito, a mais citada foi por meio de método próprio $(82,1 \%)$.

Dos farmacêuticos que justificaram o ato de não prescrever, os principais motivos foram por considerar que não faz diferença em seu dia a dia $(36,5 \%)$, por não se sentirem preparados $(14,9 \%)$ e também pelo fato do paciente não solicitar $(8,1 \%)$ (Figura 1). 


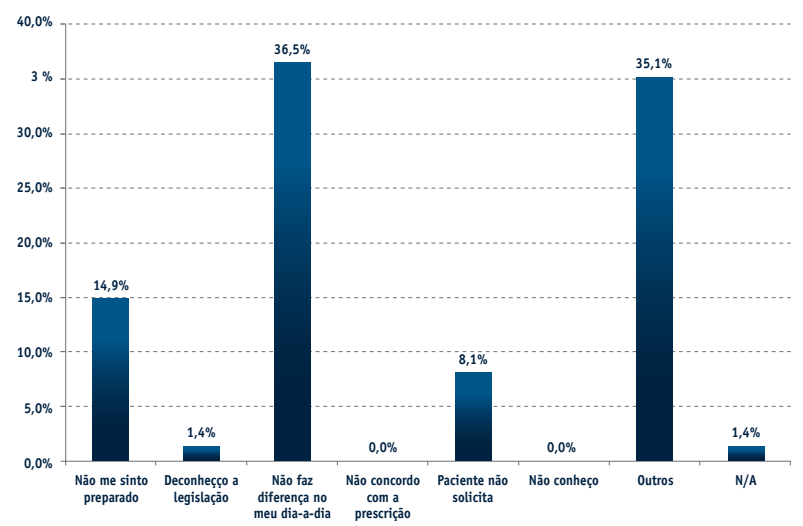

Figura 1: Motivos para não realizarem a prescrição farmacêutica, de acordo com os farmacêuticos responsáveis pelas farmácias de dispensação no município de Maringá, PR, Brasil, no período de $12 / 2014$ a 01/2015

Quanto aos $38(34,0 \%)$ profissionais farmacêuticos que afirmaram estar prescrevendo, $26(68,4 \%)$ não possuíam um formulário de prescrição específico. Somado a isso, dos 121 farmacêuticos que não prescreveram e não leram as resoluções referentes a este assunto, 96 (79,3\%) realizavam a indicação farmacêutica.

Com relação ao questionamento se os farmacêuticos se consideravam atualizados a respeito das classes farmacêuticas, como medicamentos novos, posologias, $119(74,8 \%)$ alegaram que sim. Na opinião dos farmacêuticos com relação ao que deveria ser feito para se capacitar, destacaram a oferta de curso de atualização pelo CRF com 52,5\% e a oferta de especialização e a autoatualização com $20,0 \%$ cada. A oferta de cursos de atualização pelas Instituições de Ensino Superior (IES) foi a menos escolhida pelos entrevistados, representando $2,5 \%$ (Figura 2)

A Tabela 3 mostra a análise de associação entre a prescrição farmacêutica com o fato do profissional farmacêutico ter ou não especialização, o tempo de formado, local onde se formou, tipo de currículo e a função que exerce na farmácia de dispensação. Os resultados mostraram que não houve associação significativa das variáveis selecionadas com relação à prescrição farmacêutica.

Os achados do estudo mostraram um cenário bastante interessante frente à prescrição farmacêutica, tema bastante recente no Brasil. As variáveis de natureza sociodemográfica, sexo feminino e faixa etária jovem dos farmacêuticos (20 a 29 anos) são convergentes com outras pesquisas realizadas em farmácias no Brasil. Em Ribeirão Preto, SP, $64 \%$ dos entrevistados eram do sexo feminino e $47 \%$ tinham idade entre 22 a 29 anos (11); em Natal, RN, $61 \%$ eram do sexo feminino e 50\% apresenta- vam idade entre 26 a 31 anos (12). E em farmácias do Estado de Santa Catarina, 68\% eram do sexo feminino (13).

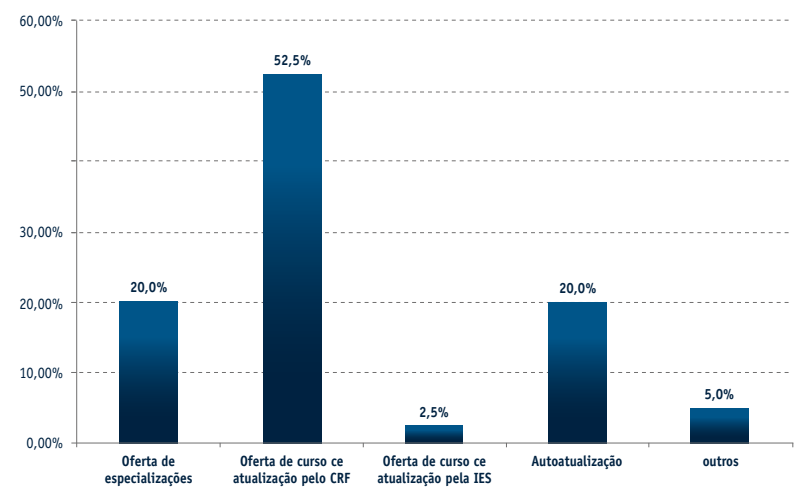

Figura 2: Formas de capacitação referidas pelos farmacêuticos responsáveis pelas farmácias de dispensação no Município de Maringá, PR, Brasil, no período de 12/2014 a 01/2015

Quanto ao tipo de medicamento que o farmacêutico pode prescrever, este trabalho evidencia que, embora a maioria dos profissionais tenha referido a leitura das Resoluções $\mathrm{N}^{\circ} 585$ e 586 de 2013, do CFF, e quase $100 \%$ terem conhecimento sobre prescrição farmacêutica, o número de profissionais que tem conhecimento sobre o que pode ser prescrito ainda é muito pequeno. Ainda, os farmacêuticos que consideraram conhecer sobre o tema, mesmo não lendo as resoluções, afirmaram que este conhecimento foi adquirido por meio de informativos e diálogos com fiscais do Conselho e colegas de trabalho.

A Resolução CFF N $N^{\circ} 586 / 2013$ destaca que o farmacêutico poderá realizar a prescrição de medicamentos e outros produtos com finalidade terapêutica, cuja dispensação não exija prescrição médica, incluindo medicamentos industrializados e preparações magistrais (alopáticos ou dinamizados) e plantas medicinais (6).

A lei que rege a comercialização de medicamentos não sujeitos à receita médica varia de acordo com o país. Na Austrália, Nova Zelândia e Finlândia há uma categoria de medicamentos, denominada pela sigla em inglês NPM (non-prescription medicines), ou seja, medicamentos não sujeitos à prescrição médica, que só podem ser vendidos mediante a intervenção e a orientação de um profissional farmacêutico. No Reino Unido, existem duas categorias de NPM: os medicamentos de venda livre e os medicamentos de farmácia, que só podem ser vendidos sob supervisão farmacêutica. Nos Estados Unidos, estes medicamentos estão disponíveis em grandes quantidades para autosseleção em prateleiras de livre acesso (14). 


\section{Angramana}

Semelhante à maioria das iniciativas em saúde, a introdução e o desenvolvimento da prescrição farmacêutica vêm com uma variedade de desafios. Uma das potenciais barreiras que impede os farmacêuticos de realizar este ato é a aceitação dos próprios profissionais farmacêuticos (15).
O elevado número de farmacêuticos que ainda realizam a indicação farmacêutica e a baixa adesão à prática da prescrição farmacêutica parece mostrar que o ato de documentar a intervenção feita na terapia farmacológica e não farmacológica intimida o profissional, mostrando que este não tem segurança para prescrever.

Tabela 3: Associação entre prescrição farmacêutica e variáveis de caracterização de farmacêuticos responsáveis pelas farmácias de dispensação no Município de Maringá, PR, no período de 12/2014 a 01/2015

\begin{tabular}{|c|c|c|c|c|}
\hline \multirow{2}{*}{ Variáveis } & \multicolumn{2}{|c|}{ Prescrição Farmacêutica } & \multirow{2}{*}{ RP (IC95\%) } & \multirow{2}{*}{$\mathbf{p}$} \\
\hline & Sim & Não & & \\
\hline \multicolumn{5}{|c|}{ Especialização } \\
\hline Sim & 13 & 34 & \multirow{2}{*}{$\begin{array}{c}0,69 \\
(0,40-1,19)\end{array}$} & \multirow{2}{*}{0,248} \\
\hline Não & 26 & 39 & & \\
\hline \multicolumn{5}{|c|}{ Tempo de formado (ano) } \\
\hline$<3$ & 7 & 18 & \multirow{3}{*}{$\begin{array}{c}0,78 \\
(0,46-1,31)\end{array}$} & \multirow{3}{*}{0,429} \\
\hline 4-6 & 8 & 22 & & \\
\hline 7 ou + & 23 & 33 & & \\
\hline \multicolumn{5}{|c|}{ Universidade onde se formou } \\
\hline Pública & 10 & 21 & \multirow{2}{*}{$\begin{array}{c}0,88 \\
(0,49-1,58)\end{array}$} & \multirow{2}{*}{0,835} \\
\hline Particular & 29 & 50 & & \\
\hline \multicolumn{5}{|c|}{ Tipo de formação } \\
\hline Habilitação & 12 & 32 & \multirow{2}{*}{$\begin{array}{c}0,69 \\
(0,39-1,21)\end{array}$} & \multirow{2}{*}{0,251} \\
\hline Generalista & 27 & 41 & & \\
\hline \multicolumn{5}{|c|}{ Função na farmácia } \\
\hline Farmacêutico auxiliar & 16 & 30 & \multirow{2}{*}{$\begin{array}{c}1,00 \\
(0,59-1,67)\end{array}$} & \multirow{2}{*}{1,15} \\
\hline Farmacêutico responsável técnico & 23 & 43 & & \\
\hline
\end{tabular}

Teste Exato de Fisher. * $\mathrm{p}<0.05$. RP= razão de prevalência. IC95\%= intervalo de confiança de $95 \%$.

Podemos inferir que esta insegurança ao prescrever, registrar por escrito uma indicação terapêutica, vem da desvalorização histórica da profissão. Com a transformação tecnológica pela qual passou o medicamento e o consequente desenvolvimento das atuais técnicas de sua comercialização, o farmacêutico ficou por anos relegado a mera função de dispensador de medicamentos, sendo muitas vezes confundidos com balconistas. $(16,17)$.

Além disso, a dispensação farmacêutica nunca foi percebida como uma atividade importante pela população e, geralmente, os pacientes nem sequer esperam o contato com o farmacêutico (18). De maneira geral, as pessoas procuram a farmácia com o intuito de comprar um produto, e não o de obter um serviço profissional qualificado, provedor de informações específicas sobre medicamentos $(19,20)$.

Este trabalho evidenciou que grande parte dos farmacêuticos que alegou estar prescrevendo, na realidade, está confundindo prescrição com indicação farmacêutica, pelo fato de não possuírem um formulário de prescrição específico. A Resolução CFF N N 586/2013 afirma que, para caracterizar uma prescrição farmacêutica, essa deverá ser redigida em vernáculo, por extenso, de modo legível, observados a nomenclatura e o sistema de pesos e medidas oficiais, sem emendas ou rasuras, devendo conter certos componentes mínimos, como a identificação do estabelecimento farmacêutico, consultório ou serviço de saúde ao qual o farmacêutico está vinculado, nome completo e contato do paciente, descrição da terapia farmacológica e não farmacológica, nome completo do farmacêutico, assinatura e número de registro no Conselho Regional de Farmácia e local e data da prescrição (6).

Além disso, o fato do profissional afirmar que a prescrição não faz diferença em seu dia-a-dia pode retratar indiferença, falta de conhecimento e de preparo do profissional com relação a este aspecto. Embora tenha sido pequena a frequência do motivo "paciente não solicita prescrição", esta situação pode ocorrer provavelmente porque a função do profissional farmacêutico é ainda muito pouco conhecida pela população. 
Em uma revisão sistemática, que abrangeu informações sobre o papel do farmacêutico na venda de medicamentos não sujeitos à prescrição médica em diversos países, foi evideciado que o fornecimento de informações e o aconselhamento sobre medicamentos por parte dos farmacêuticos nem sempre é suficiente e de qualidade, sendo necessária a busca por métodos para desenvolver e para melhorar a interação deste profissional com o paciente, no momento que este solicite um atendimento em busca de um medicamento isento de prescrição médica (14).

Com relação à análise de associação, as variáveis especialização, tempo de formado, local onde se formou, tipo de currículo e a função que exerce na farmácia não apresentaram associação significativa com o ato de realizar ou não a prescrição farmacêutica. Contudo, esperava-se que o fato de um farmacêutico ter mais tempo de formado ou ter uma responsabilidade maior na farmácia, por ser o responsável técnico, daria a ele maior segurança e confiança durante a prescrição.

Um levantamento realizado no Reino Unido mostrou que os farmacêuticos com mais de 5 anos de experiência ou que trabalham em equipes são mais propensos a praticar a prescrição independente (21). Um possível motivo para que nossos profissionais mais experientes não realizem a prescrição pode ser em virtude de não ser uma prática usual até o momento, devido à recente regulamentação da atividade.

A variável especialização não mostrou associação significativa e pode ser justificada também devido à prescrição farmacêutica ser regulamentada em uma resolução relativamente nova, de 2013. Ou seja, não houve tempo hábil ainda para que as instituições de ensino incluíssem a prescrição em seus currículos, bem como as especializações que abordam esse tema estão sendo desenvolvidas e ainda não formaram profissionais adequadamente preparados.

Outro dado que não mostrou associação com a prescrição foi o local de formação, sendo que, independente da universidade ser pública ou particular, a ação do farmacêutico como prescritor ainda não está sendo valorizada nos currículos. Acredita-se que, devido à precocidade do tema, este será um assunto de muitos debates nas instituições de ensino.

Nesse sentido, vale ressaltar que o tipo de currículo no qual o farmacêutico se formou poderia ter resultado em uma associação significativa, já que, desde 2002, com a introdução das DCN (Diretrizes Curriculares Nacionais) e do currículo generalista, o profissional farmacêutico deveria ser formado com habilidades que incluam a responsabilidade e a atenção à saúde do paciente, a tomada de decisão, a comunicação, liderança, a educação permanente, entre outras (22).

No entanto, o que é observado atualmente, parece ser uma formação muito tecnicista dos farmacêuticos em detrimento às outras habilidades, principalmente nas que envolvem relacionamento com os usuários. Nossos cursos priorizam o desenvolvimento de técnicas para a realização de serviços nas mais diversas áreas de atuação do profissional e pouco enfocam o desenvolvimento humanista, crítico e reflexivo para a perfeita relação farmacêutico-paciente. Somente uma formação com foco na saúde integral do paciente poderá levar os profissionais a atuarem no verdadeiro cuidado farmacêutico. Tradicionalmente, como visto em outros países, enquanto os farmacêuticos são formados e treinados na seleção e gestão de medicamentos sujeitos à receita médica, o papel deles na prática clínica e, consequentemente, na prescrição tem sido limitado (23).

Durante a coleta dos dados, foi percebida certa resistência dos profissionais a participarem da pesquisa, como também em algumas farmácias houve a necessidade de se retornar mais de uma vez ao estabelecimento, pois o farmacêutico, naquele momento, não se fazia presente.

É importante ressaltar a dificuldade em discutir os resultados desta pesquisa com a literatura, considerando a escassez ou quase inexistência de publicações atuais que abordam este tema. Ações conjuntas entre a academia e entidades de classe devem acontecer com o intuito de ampliar as discussões e esclarecimentos na formação e na prática dos profissionais, considerando a Resolução $N^{\circ}$ 586/2013 que encerra a concepção de prescrição como a ação de recomendar algo ao paciente, bem como esclarecimentos à população, para que este ato seja valorizado e colocado em prática.

\section{CONCLUSÃO}

Os resultados obtidos levaram à conclusão de que a prescrição farmacêutica é bem entendida na sua teoria, entretanto é incipiente na prática por ser um assunto ainda novo em termos de legislação, sendo também pouco valorizada pelos farmacêuticos em sua atividade profissional.

\section{AGRADECIMENTOS:}

À Universidade Estadual de Maringá e ao Conselho Regional de Farmácia do Paraná. 


\section{REFERÊNCIAS}

1. Novaes MRG, Lolas F, Sepúlveda AQ. Ética e Farmácia: Uma abordagem latinoamericana em saúde. Brasília: Thesaurus. 2009.

2. Reed E. Contribuições do ensino de química farmacêutica para formação do profissional farmacêutico. [Tese]. Brasília: Universidade de Brasília, Brasília. 2000.

3. Menezes EBB. Atenção farmacêutica em xeque. Rev. Pharm. Brasileira 2000; 22: 28.

4. Costa MA. Vanguarda, humanidade, aprendizado. Rev. Pharm. Brasileira 2001; 26: 28.

5. BRASIL. Conselho Federal de Farmácia. Resolução RDC n $n^{\circ} 585$, de 29 de agosto de 2013. Regulamenta as atribuições clínicas do farmacêutico e dá outras providências. Diário Oficial da União, 25 de agosto de 2013. Seção 1. p. 186.

6. BRASIL. Conselho Federal de Farmácia. Resolução RDC $n^{\circ} 586$, de 29 de agosto de 2013. Regula a prescrição farmacêutica e dá outras providências. Diário Oficial da União, 26 de agosto de 2013. Seção 1. p. 136.

7. BRASIL. Conselho Federal de Farmácia. Resolução RDC n ${ }^{\circ} 357$, de 20 de abril de 2001. Aprova o regulamento técnico das Boas Práticas de Farmácia. Diário Oficial da União, 27 de abril de 2001. Seção 1. p. 24-30.

8. Poh EW, McArthur A, Roughead L. The effects of pharmacist prescribing on patient outcomes in the hospital setting: a systematic review protocol. JBI Database Syst Rev Implem Rep 2015; 13(12):4-13. DOI 10.11124/jbisrir-2015-2411

9. IBGE. Instituto Brasileiro de Geografia e Estatística. Banco de dados dos municípios brasileiros, 2004.

10. Bussab W, Morettin PA. Estatística Básica. São Paulo: Saraiva. 2004.

11. Silva LR. Conhecimentos e atitudes dos farmacêuticos sobre regulamentação da profissão e funcionamento de drogarias - Uma abordagem sanitária. [Dissertação]. Ribeirão Preto: Faculdade de medicina, São Paulo. 2002.

12. Souza SS. Farmacêuticos e suas atividades em farmácias comunitárias: uma análise de perfil. [Dissertação]. Rio Grande do Norte: Universidade Federal do Rio Grande do Norte, Natal. 2012.

13. França Filho JB, Correr CJ, Rossignoli P, Melchiors AC, Fernández-Llimós F, Pantarolo R. Perfil dos farmacêuticos e farmácias em Santa Catarina: indicadores de estrutura e processo. Rev. Bras. Cienc. Farm. 2008; 44(1): 105-113.

14. Eikenhorst LV, Salema NE, Anderson C. A systematic review in select countries of the role of the pharmacist in consultations and sales of non-prescription medicines in community pharmacy. Res Social Adm Pharm 2016; 1-22. E-pub. DOI http://dx.doi.org/10.1016/j.sapharm.2016.02.010.

15. Bourne RS, Baqir W, Onatade R. Pharmacist independent prescribing in secondary care: opportunities and challenges. Int J Clin Pharm 2016; 38:1-6. DOI 10.1007/ s11096-015-0226-9.

16. Hepler, CD, Strand LM. Opportunities and responsibilities in pharmaceutical care. Am J. Hosp Pharm, 1990; 47: 533-543.

17. Peretta MD, Ciccia G. Reengenharia farmacêutica: guia para implementar a atenção farmacêutica. Brasília: Ethosfarma. 2000.

18. Pepe VLE, Castro CGSO. A interação entre prescritores, dispensadores e pacientes: informação compartilhada como possível benefício terapêutico. Cad. Saúde Pública 2000; 16(3): 815-22.

19. Hassell K, Rogers A, Noyce P. Community pharmacy as a primary health and self-care resource: a framework for understanding pharmacy utilisation. Health Soc Care Community 2000; 8(1): 40-49.

20. Hibbert D, Bissell P, Ward PR. Consumerism and professional work in the community pharmacy. Soc Health Illness. 2002; 24(1): 46-65. DOI 10.1111/1467-9566.00003.

21. Bourne RS, Whiting P, Brown LS, Borthwick M. Pharmacist independent prescribing in critical care: results of a national questionnaire to establish the 2014 UK position. Int J Pharm Pract 2016.;24 (2):104-113. DOI 10.1111/ijpp.12219.

22. BRASIL. Conselho Nacional de Educação. Resolução RDC $n^{\circ}$ 2, de 19 de fevereiro de 2002. Institui Diretrizes Curriculares Nacionais do Curso de Graduação em Farmácia. Diário Oficial da União, 4 de março de 2002. Seção 1. p. 9.

23. Law MR, Ma T, Fisher J, Sketris IS. Independent Pharmacist Prescribing in Canada. Can Pharm J 2012; 145(1): 17-23. DOI 10.3821/1913-701X-145.1.17. 